\title{
Copy Number Heterogeneity in the Virulence Plasmid of Salmonella enterica
}

\author{
María A. Sánchez-Romero*, Ángela Mérida-Floriano and Josep Casadesús* \\ Departamento de Genética, Facultad de Biología, Universidad de Sevilla, Sevilla, Spain
}

Quantitative PCR analysis shows that the virulence plasmid of Salmonella enterica serovar Typhimurium (pSLT) is a low-copy-number plasmid, with 1-2 copies per chromosome. However, fluorescence microscopy observation of pSLT labeled with a lacO fluorescent tag reveals cell-to-cell differences in the number of foci, which ranges from 1 to 8 . As each focus must correspond to $\geq 1$ plasmid copy, the number of foci can be expected to indicate the minimal number of pSLT copies per cell. A correlation is found between the number of foci and the bacterial cell volume. In contrast, heterogeneity in the number of foci appears to be independent of the cell volume and may have stochastic origin. As a

OPEN ACCESS

Edited by:

Eun-Jin Lee,

Korea University, South Korea

Reviewed by: Christopher Morton Thomas, University of Birmingham, United Kingdom Francisco P. Chávez, University of Chile, Chile

*Correspondence: Josep Casadesús casadesus@us.es

María A. Sánchez-Romero mtsanchez@us.es

Specialty section: This article was submitted to Infectious Diseases, a section of the journal Frontiers in Microbiology

Received: 28 August 2020 Accepted: 18 November 2020 Published: 04 December 2020

Citation: Sánchez-Romero MA, Mérida-Floriano Á and

Casadesús J (2020) Copy Number Heterogeneity in the Virulence Plasmid of Salmonella enterica.

Front. Microbiol. 11:599931. doi: 10.3389/fmicb.2020.599931 consequence of copy number heterogeneity, expression of a pSLT-bone reporter gene shows high levels of cell-to-cell variation, especially in actively dividing cultures. These observations support the notion that low-copy-number plasmids can be a source of gene expression noise in bacterial populations.

Keywords: Salmonella, virulence plasmid, copy number, phenotypic heterogeneity, noise

\section{INTRODUCTION}

Plasmid-encoded genes of bacterial pathogens can influence multiple aspects of the pathogenhost interaction, such as adhesion to host surfaces, invasion of host cells, intracellular survival, colonization of organs, and modulation of host immune responses (Pilla and Tang, 2018). In Salmonella enterica, plasmids that encode virulence determinants were described several decades ago (Gulig et al., 1993). More recent studies have identified plasmid-borne genes that control the expression of chromosomal loci such as the flagellar network and genes encoding efflux pumps (Huttener et al., 2019; Lian et al., 2019). An unsuspected finding is the existence of Salmonella plasmids that harbor essential genes (Canals et al., 2019). The Salmonella plasmidome is also a massive reservoir of antimicrobial resistance determinants (Emond-Rheault et al., 2020).

In serovar Typhimurium, most strains carry a plasmid of 50-100 kb known as the Salmonella virulence plasmid (Gulig et al., 1993; Guiney et al., 1994; Baumler et al., 1998). This plasmid was designated virulence plasmid of $S$. enterica serovar Typhimurium (pSLT) in strain LT2 (Jones et al., 1982), and the acronym was later extended to sibling plasmids of other Salmonella Typhimurium strains (Rotger and Casadesus, 1999).

Plasmid pSLT belongs to the $\mathrm{MOB}_{\mathrm{F} 1}$ group on the basis of relaxase classification (GarcillanBarcia et al., 2009) and harbors genes for conjugal transfer (tra) homologous to those of the $\mathrm{F}$ sex factor (Garcia-Quintanilla and Casadesus, 2011); however, tra gene deletions are relatively common (Rotger and Casadesus, 1999). All Salmonella virulence plasmids share a $\sim 8 \mathrm{~kb}$ region, Salmonella plasmid virulence $(s p v)$, required for bacterial proliferation in the phagocytic system of warm-blooded vertebrates (Gulig et al., 1993; Rotger and Casadesus, 1999). Additional pSLT 
genes involved in pathogenesis are the pef fimbrial operon (Baumler et al., 1996), the rck gene which confers resistance to complement killing (Heffernan et al., 1992) and promotes epithelial cell invasion (Cirillo et al., 1996), and the rsk gene, involved in resistance to host complement (Vandenbosch et al., 1989). Hybrid plasmids harboring virulence and antibiotic resistance determinants have been described in S. enterica clinical isolates (Guerra et al., 2002; Mendoza et al., 2009).

Plasmid pSLT has a low $(\geq 1)$ copy number per cell (Camacho et al., 2005), and possesses at least three maintenance mechanisms: control of replication and copy number mediated by homologs of F plasmid RepB and RepC (Tinge and Curtiss, 1990), a canonical partition system, parAB (Tinge and Curtiss, 1990), and two toxin-antitoxin systems, $c c d A B S T$ and $v a p B C 2_{\mathrm{ST}}$ (LobatoMarquez et al., 2015). Co-operative activity of such systems may explain the remarkable stability of pSLT, whose frequency of spontaneous curing is around $10^{-7}$ per cell and generation under laboratory conditions (Garcia-Quintanilla et al., 2006).

In this study, we have used fluorescence microscopy and flow cytometry to track pSLT in individual S. enterica cells. We provide evidence that the pSLT copy number is heterogeneous, especially among dividing cells. Tight control of pSLT replication and partition (Lobato-Marquez et al., 2015) is thus compatible with cell-to-cell differences that produce gene expression noise at the population level. We discuss the possibility that copy number heterogeneity might be an adaptive trait.

\section{MATERIALS AND METHODS}

\section{Bacterial Strains, Plasmids, and Growth Conditions}

Salmonella enterica strains used in this study belong to serovar Typhimurium and derive from ATCC 14028 (Table 1). For construction of strain SV8214, a lacO cassette consisting of 48 repeats of the lactose operator was integrated into the tra operon of the virulence plasmid. In the initial step of pSLTlacO construction, the lacO array was PCR-amplified from p48LacO, a derivative of pLAU07 (Lau et al., 2003) obtained from David Sherratt's laboratory (University of Oxford, England). Amplification was achieved using primers lacO1 and lacO2 (Supplementary Table S1), and the amplified fragment was cloned onto pDOC-K (Lee et al., 2009), generating plasmid pIZ2031 (Table 2). The prefix pIZ was registered at the Plasmid Reference Center in 1986 (Lederberg, 1986). The lacO array and the kanamycin resistance cassette were inserted into pSLT using the lambda Red recombination system (Datsenko and Wanner, 2000) and primers finO1 and finO2 (Supplementary Table S1). Plasmid pWX17, obtained also from David Sherratt's laboratory, is a pUC18 derivative that carries the yellow fluorescent protein $(y f p)$ gene under the control of an arabinose-dependent promoter (Wang et al., 2005). Introduction of pWX17 into appropriate strains was achieved by transformation. Induction of the arabinose-dependent promoter was achieved with $0.02 \% \mathrm{~L}$-arabinose (Sigma-Aldrich, St. Louis, Missouri). Bacterial cultures were grown in Bertani's lysogeny broth (LB) at $37^{\circ} \mathrm{C}$ with shaking.

\section{Determination of Plasmid Copy Number by Quantitative PCR}

DNA extraction was performed using a method that does not modify the chromosome/plasmid DNA ratio (Brandi et al., 2000). Aliquots of crude cell extracts were diluted and subjected to quantitative PCR to estimate the relative content of chromosomal and plasmid DNA. Approximately, $10 \mathrm{ng}$ total DNA were used for each amplification. Quantitative RT-PCR reactions were performed in a Light Cycler 480 II apparatus (Roche). Real-time cycling conditions were as described elsewhere (Camacho et al., 2005). Primers (Supplementary Table S1) were designed with Primer3Plus software. ${ }^{1}$

\section{In vivo Visualization of pSLT by Fluorescence Microscopy}

Strains were grown at $37^{\circ} \mathrm{C}$ in $\mathrm{LB}$ and diluted to $1: 100$ in fresh medium. Samples of $1.5 \mathrm{ml}$ were collected by centrifugation at $3,400 \times g$ for $5 \mathrm{~min}$ at different optical densities $\left(\mathrm{OD}_{600}\right)$ along the growth cycle. YFP-LacI was induced for $30 \mathrm{~min}$ with $0.02 \%$ L-arabinose. Cells were stained with $1 \mu \mathrm{g} / \mathrm{ml}$ FM4-64 and $5 \mu \mathrm{g} / \mathrm{ml}$ Hoechst 33258 in $10 \mu \mathrm{l}$ of mounting medium (40\% glycerol in $0.02 \mathrm{M} \mathrm{PBS}, \mathrm{pH} 7.5)$ and fixed onto a poly-lysine-treated coverslip. Images were obtained by using an Olympus IX-70 Delta Vision fluorescence microscope equipped with a 100X UPLS Apo Objective. Pictures were taken using a CoolSNAP HQ/ICX285 camera and analyzed using ImageJ software (Wayne Rasband, Research Services Branch, Maryland).

\section{Flow Cytometry}

Strain SV8960 was grown at $37^{\circ} \mathrm{C}$ in $\mathrm{LB}$. At appropriate times, samples of $1.5 \mathrm{ml}$ were collected by centrifugation, washed, and re-suspended in phosphate-buffered saline (PBS) for flow cytometry analysis. Data acquisition was performed using a Cytomics FC500-MPL cytometer (Beckman Coulter,

TABLE 1 | Salmonella enterica strains constructed for this study.

\begin{tabular}{|c|c|}
\hline Strain & Relevant traits \\
\hline SV8214 & $\mathrm{pSLT} 48 \times \mathrm{lacO}\left(\mathrm{Km}^{\mathrm{P}}\right)$ \\
\hline SV8216 & pSLT/pWX17 (lacl::YFP) \\
\hline SV8217 & $\mathrm{pSLT} 48 \times 1 \mathrm{lacO}\left(\mathrm{Km}^{\mathrm{P}}\right) / \mathrm{pWX} 17$ (lacl::YFP) \\
\hline SV8398 & pSLT $48 \times$ lacO $\triangle$ parA::Km²/pWX17 (lacl::YFP) \\
\hline SV8960 & pSLT-GFP (constitutive gfp gene inserted at the traA locus; $\mathrm{Km}^{\mathrm{R}}$ \\
\hline
\end{tabular}

TABLE 2 | Plasmids used in strain constructions.

\begin{tabular}{lll}
\hline Name & Relevant traits & Reference or source \\
\hline pDOC-K & Kanamycin resistance cassette $\left(\mathrm{Km}^{\mathrm{R}}\right)$ & Lee et al., 2009 \\
p48LacO & 48 lac operator array & Lau et al., 2003 \\
plZ2031 & pDOC-K-48XlacO $\left(\mathrm{Km}^{\mathrm{R}} \mathrm{Ap} \mathrm{p}^{\mathrm{R}}\right)$ & This study \\
pWX17 & lacl::YFP $\left(\mathrm{Ap}^{\mathrm{R}}\right)$ & Garcia-Quintanilla et al., 2006
\end{tabular}

${ }^{1}$ http://www.bioinformatics.nl/cgi-bin/primer3plus/primer3plus.cgi/ 
Brea, California), and data were analyzed with FlowJo X, version 10.0.7r software (Tree Star, Oregon).

\section{Determination of Bacterial Cell Volume}

Micrographs of phase-contrast images were used to measure the length and the width of the cells using ImageJ software. Volumes of bacterial cells were calculated as described elsewhere (Volkmer and Heinemann, 2011). For each condition, the dimensions of 150-300 individual cells were measured.

\section{Serum Treatment}

Overnight cultures grown at $37^{\circ} \mathrm{C}$ in $\mathrm{LB}$ were diluted to $1: 100$ in fresh medium and incubated at $37^{\circ} \mathrm{C}$ with $200 \mathrm{rpm}$ shaking for $1 \mathrm{~h}$. Cultures were split into two and mouse serum (Sigma) was added to one of the cultures to reach a final concentration of $8 \%$. Samples of $1.5 \mathrm{ml}$ were collected at different optical densities $\left(\mathrm{OD}_{600}\right)$, and treated as described above for visualization by fluorescence microscopy.

\section{Statistical Analysis}

Averages, medians, SDs, and interquartile ranges in the number of pSLT foci were calculated upon examination of 150-300 cells. The exact numbers of cells are indicated in the figure legends. A robust coefficient of variation ( $\mathrm{rCV}$ ) was obtained by dividing the interquartile range by the median of green fluorescent protein (GFP) fluorescence intensity, a parameter calculated with FlowJo X software (Shapiro, 2003).

\section{RESULTS}

\section{Determination of Plasmid Copy Number by Quantitative PCR}

The copy number of plasmid pSLT was determined using a quantitative PCR protocol. Oligonucleotide primers were designed to amplify a gene located relatively close to the origin of replication of the $S$. enterica chromosome $(\operatorname{arc} A)$, a gene located near the replication terminus ( $h i s D$ ), and the traJ and $c c d B$ genes of pSLT. The PCR efficiency (E) for each primer was calculated from amplification slopes. They were found to be similar $(-0.44<-\log \mathrm{E}<-0.39)$, thereby allowing the comparison of results. Threshold cycles were normalized, indicating the relative copy number of pSLT per chromosome. DNA samples were obtained at different growth stages in LB broth, and amplifications were performed in triplicate.

Amplification of pairwise combinations of chromosomal and plasmid-borne genes provided an estimate of the relative number of pSLT copies per chromosome. Such numbers ranged from 0.92 to 1.67 pSLT copies per chromosome (Table 3), with an average of 1.35 plasmid copies per chromosome equivalent. The SDs obtained with different gene pairs and at different growth stages were small, suggesting that the average number of PSLT copies per chromosome remained fairly constant throughout growth. However, this analysis left open the possibility that cells with higher numbers of pSLT copies were overlooked, especially if such cells were rare.
TABLE 3 | Number of plasmid of Salmonella enterica serovar Typhimurium (pSLT) copies per chromosome, determined by qPCR.

\begin{tabular}{|c|c|c|c|c|}
\hline Time & $\operatorname{traJ} / \operatorname{arcA} A^{a}$ & traJ/his $D^{b}$ & $\mathrm{ccdB} / \operatorname{arcA}^{\mathrm{a}}$ & ccdB/hisD ${ }^{b}$ \\
\hline 0 & $1.12 \pm 0.23$ & $1.27 \pm 0.32$ & $1.21 \pm 0.12$ & $1.39 \pm 0.08$ \\
\hline 60 & $0.92 \pm 0.19$ & $1.06 \pm 0.24$ & $1.03 \pm 0.31$ & $1.16 \pm 0.12$ \\
\hline 120 & $1.03 \pm 0.28$ & $1.55 \pm 0.18$ & $1.17 \pm 0.34$ & $1.48 \pm 0.32$ \\
\hline 240 & $1.29 \pm 0.11$ & $1.20 \pm 0.35$ & $1.10 \pm 0.26$ & $1.67 \pm 0.29$ \\
\hline
\end{tabular}

${ }^{a}$ Averages and $S D$ s from five independent experiments.

${ }^{b}$ Averages and $S D$ s from three independent experiments.

\section{Visualization of pSLT in Live Cells}

Labeling of plasmid pSLT was performed with the Fluorescent Repressor Operator System (FROS; Robinett et al., 1996; Lau et al., 2003), and in vivo visualization was achieved by fluorescence microscopy. For this purpose, strain SV8217 was grown overnight at $37^{\circ} \mathrm{C}$ in $\mathrm{LB}$, diluted to $1: 100$ in fresh medium, and grown to an $\mathrm{OD}_{600}$ of $0.1-0.2$. Under these conditions, fluorescence foci formed by the virulence plasmid were visualized (Figure 1A). The majority of cells had either one focus $(28 \%)$ or two $(38 \%)$. About $22 \%$ of cells had three foci and $4 \%$ had four foci (Figure 1B). Cells containing five or more foci were detected at lower frequencies (Figure 1B). As each focus must correspond to one or more plasmid copies, the number of foci can be expected to indicate the minimal number of PSLT copies per cell.

We also examined the subcellular localization of pSLT in cells containing different numbers of foci per cell. In cells containing one focus, it was located at or near mid-cell. In cells containing two foci, they were located at quarter-cell positions. In cells containing three foci, one was located near mid-cell and the other two were located at or near $1 / 4$ and $3 / 4$ positions. When cells contained four foci, these were located at $1 / 8,3 / 8,5 / 8$, and $7 / 8$ subcellular positions. A visual summary of these observations is shown in Figure 1C, plotting the position of each focus against the fraction of cell length. We can thus conclude that PSLT is not randomly positioned in the cells, and that its localization pattern depends on the number of foci per cell. Similar localization patterns have been observed in other low-copy-number plasmids (Gordon et al., 1997; Niki and Hiraga, 1997).

To test whether the subcellular position of PSLT was determined by its partition machinery, we disrupted the parA gene, which is known to encode partition functions (Tinge and Curtiss, 1990; Cerin and Hackett, 1993). The pSLT derivative lacking the parA gene was detected in spaces not occupied by nucleoids (e.g., near one cell pole; Figure 2). A pattern of this kind is typical upon Par system inactivation in low-copynumber plasmids (Niki and Hiraga, 1997).

\section{Heterogeneity of pSLT Copy Number Along the Growth Cycle}

The number of plasmid foci per cell was monitored at different stages of the growth cycle. For this purpose, an overnight culture of strain SV8217 was diluted to 1:100 in fresh LB and grown at $37^{\circ} \mathrm{C}$. Samples were periodically taken for cell visualization by fluorescence microscopy. The proportion of cells containing different numbers of fluorescent foci was found 
A
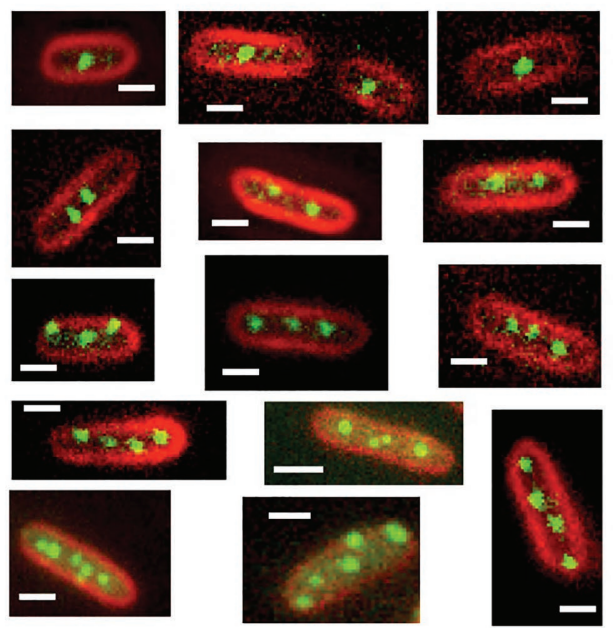

B

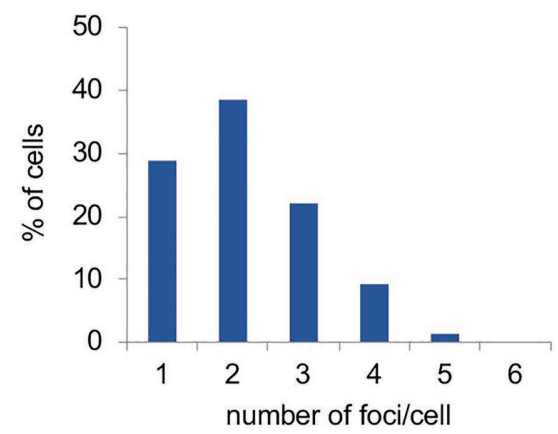

C

c

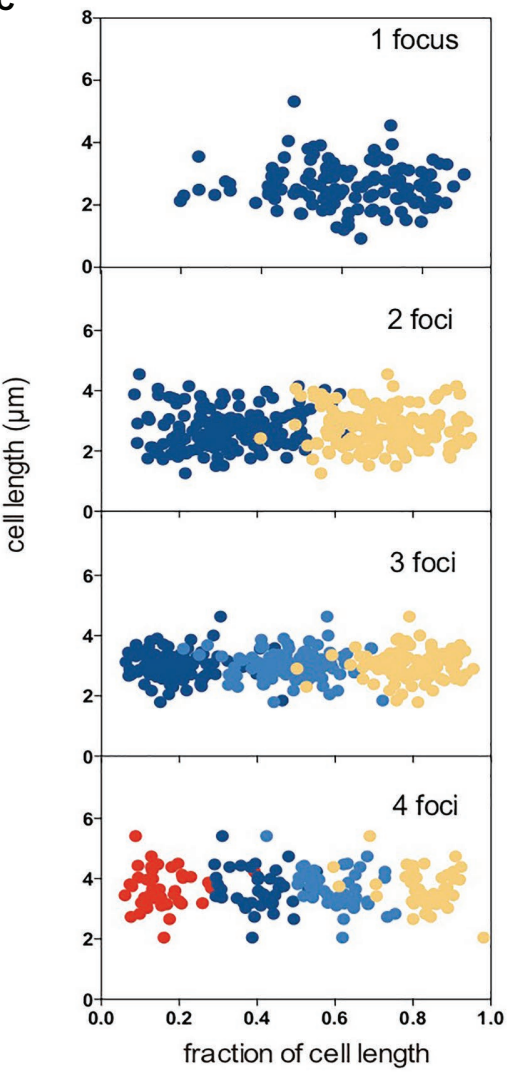

FIGURE 1 | Visualization of the pSLT plasmid tagged with YFP-LacO. (A) Fluorescence micrographs of selected cells containing pSLT-LacO plasmids. Cell membranes were stained with FM4-64 (red). YFP-tagged plasmids are visualized as green foci. (B) Graphs show cells containing one (28.8\%), two (38.5\%), three (22.0\%), four (9.2\%), and five (1.3\%). pSLT-LacO fluorescence foci per cell. The number of cells analyzed was 550. (C) Subcellular distribution of YFP-tagged pSLT-LacO as a function of cell length in cells containing 1-4 foci. A total of 444 cells were analyzed. Scale bars indicate $1 \mu$ m.

to vary along the growth curve, and up to eight foci per cell were found in actively dividing cultures (Figure 3A; Supplementary Figure S1). In contrast, the proportion of cells containing only one focus at mid-exponential phase was only 5\% (Figure 3A). Average foci number are shown in Figure 3B, together with SDs that provide quantitative assessment of cellto-cell heterogeneity in the number of foci.

The number of pSLT foci was also monitored in the presence of mouse serum, a laboratory condition that mimics conditions encountered by S. enterica inside animals (Murray et al., 2005). Choice of serum to monitor virulence-related conditions was also based upon the fact that two pSLT genes, $r s k$ and $r c k$, contribute to serum resistance (Vandenbosch et al., 1989; Heffernan et al., 1992). Cell-to-cell variation in the number of pSLT foci was detected, with numbers ranging from 1 to 5 foci during early and late exponential growth, and from 1 to 8 foci in mid exponential phase (Supplementary Table S2). These reductionist trials provide evidence that cell-to-cell variation in pSLT copy number may occur during infection.
To investigate a potential correlation between the number of plasmid foci and the bacterial cell volume, we measured the volume of $S$. enterica cells containing different numbers of foci. Volume measurements were done at different growth stages, and the results are shown in Figure $\mathbf{4}$ and Supplementary Figure S2. A clear-cut correlation was found between the average number of foci and the volume of the bacterial cell. In contrast, a correlation between the degree of heterogeneity in the number of foci and the cell volume was not detected, suggesting that plasmid copy number heterogeneity may be independent of the cell volume.

\section{Effect of pSLT Copy Number Variation on Gene Expression Heterogeneity}

Differences in gene dosage caused by copy number heterogeneity can be expected to produce cell-to-cell differences in the expression of plasmid-borne genes. To test this prediction, we cloned a constitutive green fluorescent protein $(g f p)$ gene on pSLT and monitored cell-to-cell variation in the GFP level using flow cytometry. Cell-to-cell variations in GFP fluorescence were higher upon active growth (Figures 5A,B). To obtain a quantitative 
MERGE
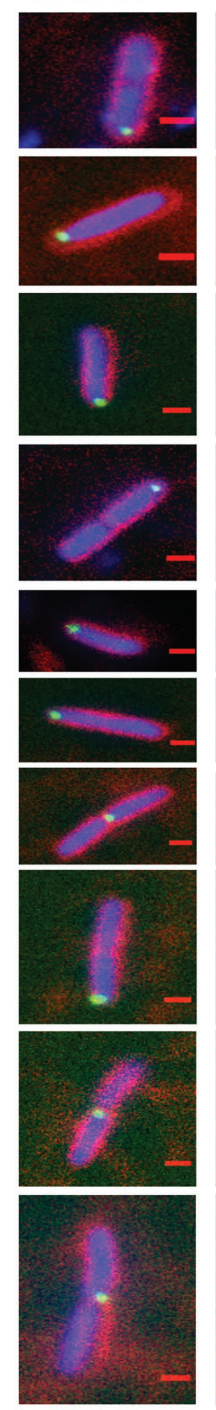

DNA
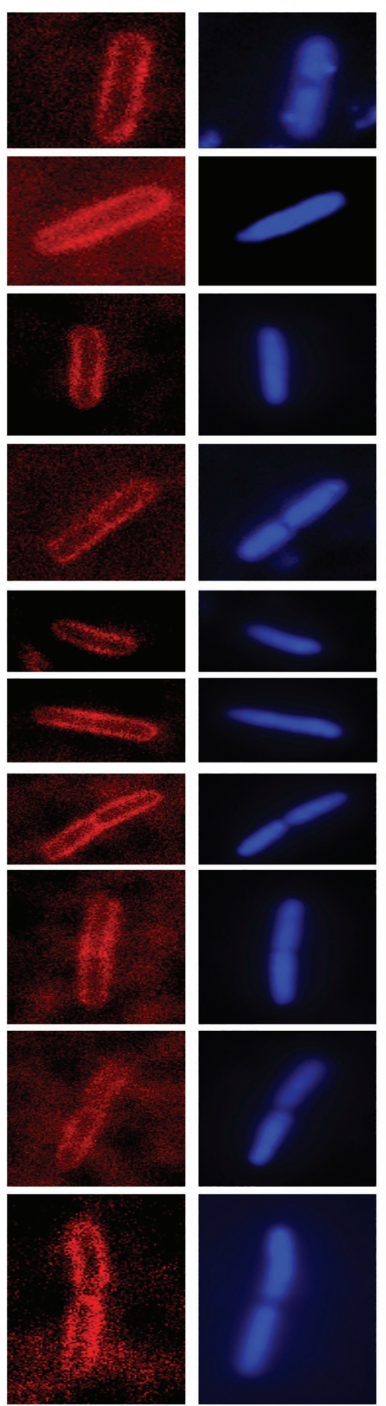
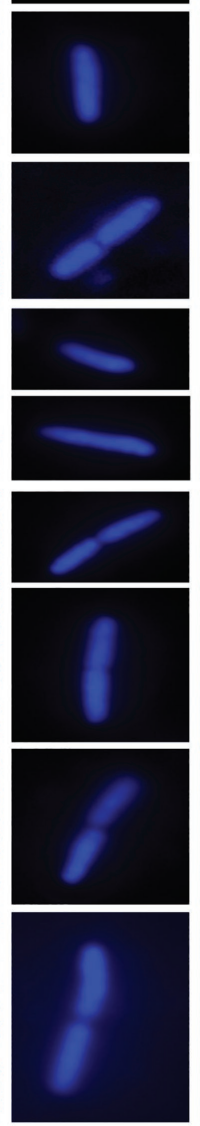

pSLT
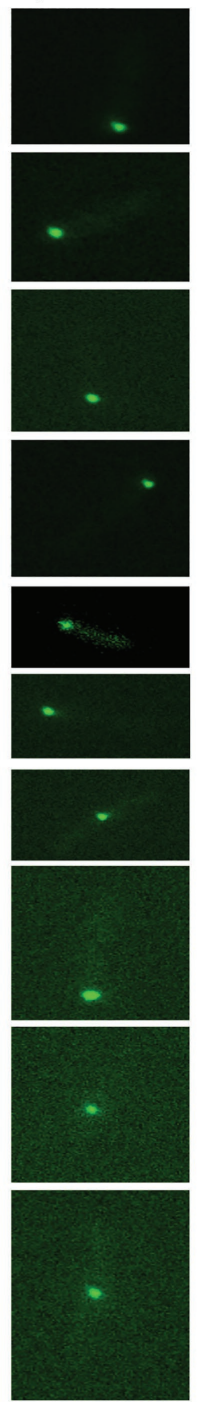

FIGURE 2 | Subcellular localization of the Salmonella virulence plasmid in a strain with a defective PSLT partitioning system. Cell membranes were stained with FM4-64 (red), DNA was stained with Hoechst 33258 (blue) and green foci revealed YFP-tagged pSLT. Scale bars indicate $1 \mu \mathrm{m}$.

estimation of the noise generated by copy number variation, rCVs were calculated. Heterogeneity in the number of foci during active growth correlates with higher noise (Figure 5C), and the higher the variation in plasmid copy number, the higher the coefficient of variation in GFP expression. This study thus suggests that pSLT-borne genes can be a source of gene expression noise due to variation in the plasmid copy number.

\section{DISCUSSION}

Quantitative PCR amplification of plasmid-borne and chromosomal DNA sequences reveals that the virulence plasmid of Salmonella enterica serovar Typhimurium, pSLT, has $\sim 1$ copy per chromosome like other F-like plasmids (Table 3). However, PCR-based calculation provides a copy number average and does not detect copy number variations among bacterial cells, especially if such cells are rare. In this study, we have exploited the FROS method (Robinett et al., 1996; Lau et al., 2003) to label the pSLT virulence plasmid with the aim of visualizing pSLT foci in individual Salmonella cells. The number of fluorescent foci indicates the minimal number of pSLT copies as partially replicated plasmids cannot be detected, and formation of plasmid aggregates is also possible.

Despite the possession of active partition systems that promote faithful distribution of one or few pSLT copies to daughter cells (Tinge and Curtiss, 1990; Lobato-Marquez et al., 2015), remarkable levels of copy number heterogeneity were detected both in LB and in the presence of mouse serum, especially in actively dividing cultures (Figures 1, 3; Supplementary Table S2). Hence, copy number heterogeneity does not seem to depend on specific growth conditions.

A correlation was found between the number of foci and the cell volume, in agreement with the classical view that plasmid replication control responds to cell volume (Chattoraj, 2000; Paulsson, 2002). In contrast, the degree of heterogeneity in the number of foci was found to be independent of the cell volume, suggesting that copy number heterogeneity may have stochastic origin. In support of this view, an analogy can be drawn between stochastic variation in the copy number of low-copy-number plasmids and other cellular processes involving low numbers of molecules (Elowitz et al., 2002; Raser and O'Shea, 2005).

As a consequence of pSLT copy number heterogeneity, the expression of a plasmid-borne $g f p$ gene showed large differences from cell to cell (Figure 5). This observation suggests that noisy gene expression of plasmid-borne genes may occur as a consequence of copy number heterogeneity. Due to a finite number effect, low-copy-number plasmids with a partitioning system may actually produce more cellular noise than random partitioning of high-copy-number plasmids (Wong et al., 2010).

At this stage, we do not know whether variation in pSLT copy number occurs also in natural environments. If that is the case, pSLT copy number heterogeneity could be expected to be frequent in animal niches in which Salmonella replicates actively such as the small intestine (Sanchez-Romero and Casadesus, 2018) and the gall bladder (Urdaneta et al., 2019). Potential advantages of phenotypic heterogeneity and concomitant noisy gene expression during Salmonella infection are thus conceivable. For instance, formation of Salmonella cells with increased dosage of conjugation proteins might contribute to the high rates of pSLT transfer detected in the murine ileum (Garcia-Quintanilla et al., 2008). High production of invasion factor Rck by a subpopulation of cells might in turn permit division of labor in a manner analogous to bistable expression of Salmonella pathogenicity island 1 (Diard et al., 2013). It is likewise conceivable that production of Pef fimbriae by a subset of cells might produce 


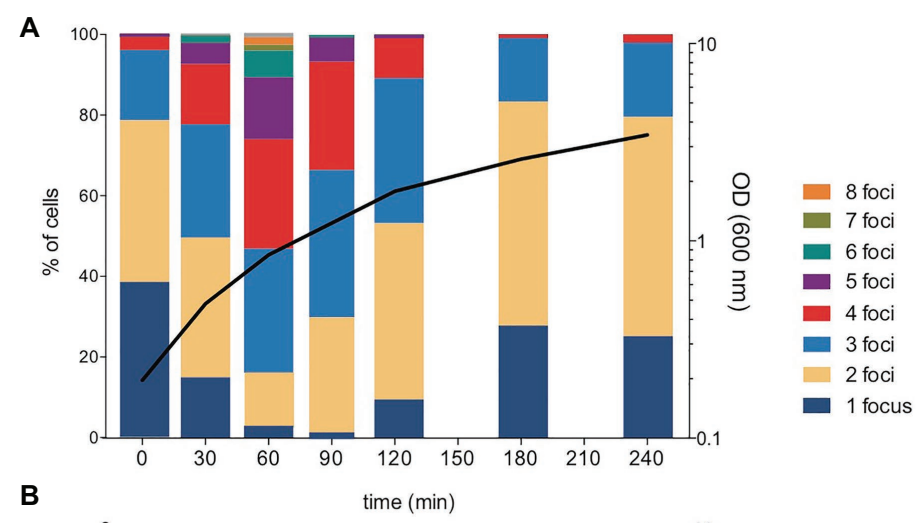

B

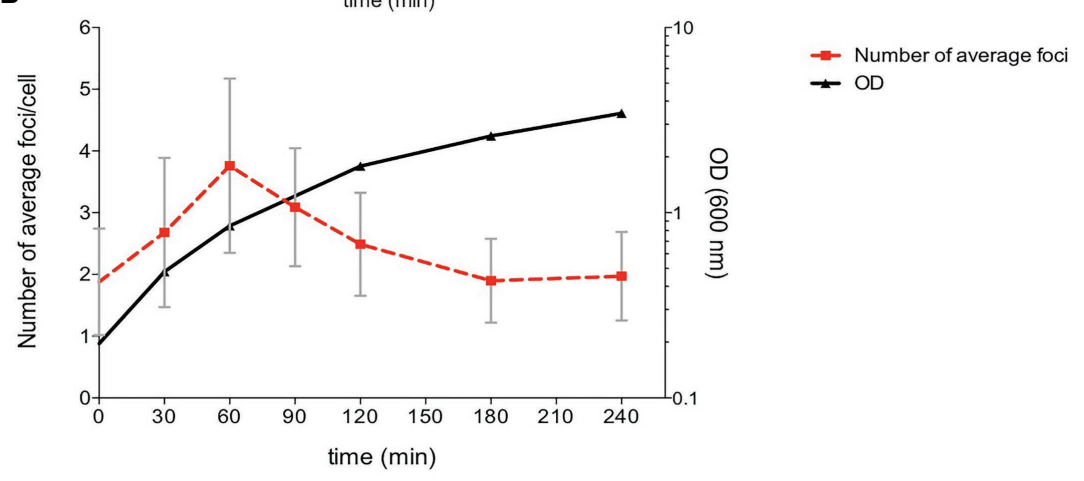

FIGURE 3 | Analysis of the number of pSLT-LacO fluorescent foci during Salmonella growth. (A) Number of pSLT foci per cell along cell growth. The proportion of cells containing different numbers of foci per cell is shown with a color code. A total of 247, 381, 272, 302, 325, 234, and 206 cells were measured at times 0 , 30 , 60, 90, 120, 180, and $240 \mathrm{~min}$, respectively. (B) Averages and SDs in the number pSLT foci per cell during active growth. Optical density at $600 \mathrm{~nm}$ is represented at the right axis in logarithmic scale.

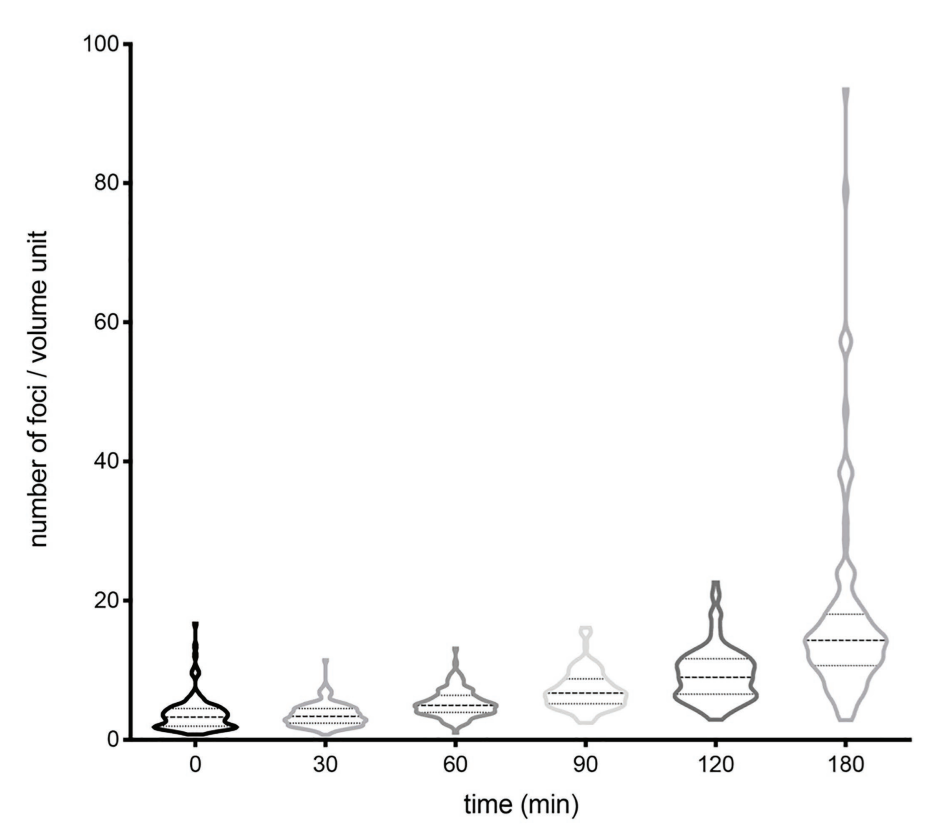

FIGURE 4 | Evolution of the number of foci per cell volume unit along the growth cycle, represented using violin plots. The number of cells analyzed at each time was 150-300. Medians and interquartile ranges are shown. The proportions of cells that fell in tails at 0, 30, 60, 90, 120, and 180 were 21.26, 22.56, 17.65, 16.10, 22.02 , and $20.39 \%$, respectively. 
A

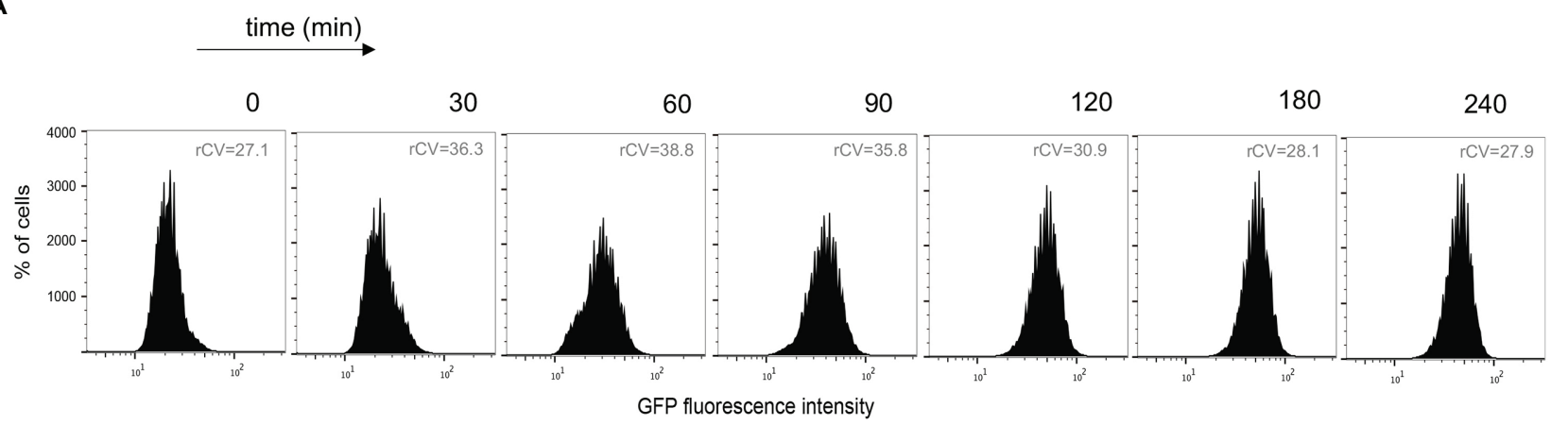

B

C
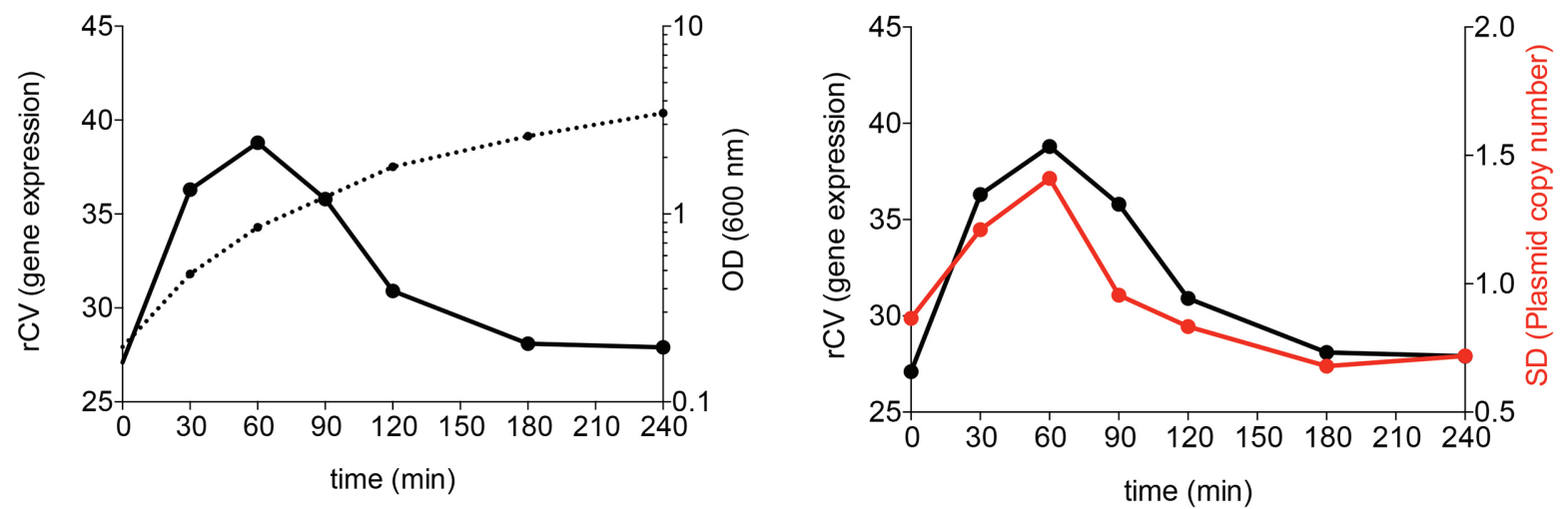

FIGURE 5 | Flow cytometry analysis of pSLT-GFP expression during growth. (A) GFP fluorescence intensity distribution in strain SV8960 (pSLT-GFP) grown in LB at $37^{\circ} \mathrm{C}$. Robust coefficients of variation (rCVs) are indicated inside graphs. (B) Analysis of the degree of heterogeneity in GFP expression along the growth cycle. The left axis displays rCVs of GFP fluorescence histograms, and the right axis represents the optical density at $600 \mathrm{~nm}$. (C) Comparative analysis of the degree of heterogeneity in GFP expression and the level of variability in the number of plasmid foci along the growth cycle. The left axis represents the robust coefficient of variation (rCV) of GFP fluorescence histograms, and the right axis represents the SD of the number of PSLT-LacO fluorescence foci per cell.

a bacterial subpopulation able to colonize specific eukaryotic surfaces, a strategy described for fimbrial systems with heterogeneous expression (Suwandi et al., 2019). Whatever the case, speculations on the adaptive value of copy number variation may be justified by the evidence that phenotypic heterogeneity is often an adaptive trait in bacterial populations (Veening et al., 2008; Casadesus and Low, 2013; Weigel and Dersch, 2018).

\section{DATA AVAILABILITY STATEMENT}

The raw data supporting the conclusions of this article will be made available by the authors, without undue reservation.

\section{AUTHOR CONTRIBUTIONS}

MS-R and ÁM-F carried out the experiments. MS-R, ÁM-F, and JC designed the experiments, interpreted results, and wrote the manuscript. All authors contributed to the article and approved the submitted version.

\section{FUNDING}

This study was supported by grant BIO2016-75235-P from the Ministerio de Ciencia e Innovación of Spain.

\section{ACKNOWLEDGMENTS}

We are grateful to Dave Sherratt for providing plasmid pWX17, to Álvaro San Millán and Rafael Peña-Miller for helpful discussions, and to Modesto Carballo, Laura Navarro, and Cristina Reyes (SGI Biología, CITIUS, Universidad de Sevilla) for help with experiments performed at the facility. We also thank the reviewers for their constructive, helpful criticism.

\section{SUPPLEMENTARY MATERIAL}

The Supplementary Material for this article can be found online at: https://www.frontiersin.org/articles/10.3389/fmicb.2020.599931/ full\#supplementary-material 


\section{REFERENCES}

Baumler, A. J., Tsolis, R. M., Bowe, F. A., Kusters, J. G., Hoffmann, S., and Heffron, F. (1996). The pef fimbrial operon of Salmonella typhimurium mediates adhesion to murine small intestine and is necessary for fluid accumulation in the infant mouse. Infect. Immun. 64, 61-68. doi: 10.1128/ IAI.64.1.61-68.1996

Baumler, A. J., Tsolis, R. M., Ficht, T. A., and Adams, L. G. (1998). Evolution of host adaptation in Salmonella enterica. Infect. Immun. 66, 4579-4587. doi: 10.1128/IAI.66.10.4579-4587.1998

Brandi, L., Falconi, M., and Ripa, S. (2000). Plasmid curing effect of trovafloxacin. FEMS Microbiol. Lett. 184, 297-302. doi: 10.1111/j.1574-6968.2000.tb0 9030.x

Camacho, E. M., Serna, A., Madrid, C., Marques, S., Fernandez, R., de la Cruz, F., et al. (2005). Regulation of fin $P$ transcription by DNA adenine methylation in the virulence plasmid of Salmonella enterica. J. Bacteriol. 187, 5691-5699. doi: 10.1128/JB.187.16.5691-5699.2005

Canals, R., Chaudhuri, R. R., Steiner, R. E., Owen, S. V., Quinones-Olvera, N., Gordon, M. A., et al. (2019). The fitness landscape of the African Salmonella Typhimurium ST313 strain D23580 reveals unique properties of the pBT1 plasmid. PLoS Pathog. 15:e1007948. doi: 10.1371/journal.ppat.1007948

Casadesus, J., and Low, D. A. (2013). Programmed heterogeneity: epigenetic mechanisms in bacteria. J. Biol. Chem. 288, 13929-13935. doi: 10.1074/jbc. R113.472274

Cerin, H., and Hackett, J. (1993). The parVP region of the Salmonella typhimurium virulence plasmid pSLT contains four loci required for incompatibility and partition. Plasmid 30, 30-38. doi: 10.1006/plas.1993.1031

Chattoraj, D. K. (2000). Control of plasmid DNA replication by iterons: no longer paradoxical. Mol. Microbiol. 37, 467-476. doi: 10.1046/j.13652958.2000.01986.x

Cirillo, D. M., Heffernan, E. J., Wu, L., Harwood, J., Fierer, J., and Guiney, D. G. (1996). Identification of a domain in Rck, a product of the Salmonella typhimurium virulence plasmid, required for both serum resistance and cell invasion. Infect. Immun. 64, 2019-2023. doi: 10.1128/IAI.64.6.2019-2023.1996

Datsenko, K. A., and Wanner, B. L. (2000). One-step inactivation of chromosomal genes in Escherichia coli K-12 using PCR products. Proc. Natl. Acad. Sci. U. S. A. 97, 6640-6645. doi: 10.1073/pnas.120163297

Diard, M., Garcia, V., Maier, L., Remus-Emsermann, M. N., Regoes, R. R., Ackermann, M., et al. (2013). Stabilization of cooperative virulence by the expression of an avirulent phenotype. Nature 494, 353-356. doi: 10.1038/ nature11913

Elowitz, M. B., Levine, A. J., Siggia, E. D., and Swain, P. S. (2002). Stochastic gene expression in a single cell. Science 297, 1183-1186. doi: 10.1126/ science.1070919

Emond-Rheault, J. G., Hamel, J., Jeukens, J., Freschi, L., Kukavica-Ibrulj, I., Boyle, B., et al. (2020). The Salmonella enterica plasmidome as a reservoir of antibiotic resistance. Microorganisms 8:1016. doi: 10.3390/microorganisms 8071016

Garcia-Quintanilla, M., and Casadesus, J. (2011). Virulence plasmid interchange between strains ATCC 14028, LT2, and SL1344 of Salmonella enterica serovar Typhimurium. Plasmid 65, 169-175. doi: 10.1016/j.plasmid.2010.12.001

Garcia-Quintanilla, M., Prieto, A. I., Barnes, L., Ramos-Morales, F., and Casadesus, J. (2006). Bile-induced curing of the virulence plasmid in Salmonella enterica serovar Typhimurium. J. Bacteriol. 188, 7963-7965. doi: 10.1128/ JB.00995-06

Garcia-Quintanilla, M., Ramos-Morales, F., and Casadesus, J. (2008). Conjugal transfer of the Salmonella enterica virulence plasmid in the mouse intestine. J. Bacteriol. 190, 1922-1927. doi: 10.1128/JB.01626-07

Garcillan-Barcia, M. P., Francia, M. V., and de la Cruz, F. (2009). The diversity of conjugative relaxases and its application in plasmid classification. FEMS Microbiol. Rev. 33, 657-687. doi: 10.1111/j.1574-6976.2009.00168.x

Gordon, G. S., Sitnikov, D., Webb, C. D., Teleman, A., Straight, A., Losick, R., et al. (1997). Chromosome and low copy plasmid segregation in E. coli: visual evidence for distinct mechanisms. Cell 90, 1113-1121. doi: 10.1016/ S0092-8674(00)80377-3

Guerra, B., Soto, S., Helmuth, R., and Mendoza, M. C. (2002). Characterization of a self-transferable plasmid from Salmonella enterica serotype Typhimurium clinical isolates carrying two integron-borne gene cassettes together with virulence and drug resistance genes. Antimicrob. Agents Chemother. 46, 2977-2981. doi: 10.1128/AAC.46.9.2977-2981.2002

Guiney, D. G., Fang, F. C., Krause, M., and Libby, S. (1994). Plasmid-mediated virulence genes in non-typhoid Salmonella serovars. FEMS Microbiol. Lett. 124, 1-9. doi: 10.1111/j.1574-6968.1994.tb07253.x

Gulig, P. A., Danbara, H., Guiney, D. G., Lax, A. J., Norel, F., and Rhen, M. (1993). Molecular analysis of $s p v$ virulence genes of the Salmonella virulence plasmids. Mol. Microbiol. 7, 825-830. doi: 10.1111/j.1365-2958.1993.tb01172.x

Heffernan, E. J., Reed, S., Hackett, J., Fierer, J., Roudier, C., and Guiney, D. (1992). Mechanism of resistance to complement-mediated killing of bacteria encoded by the Salmonella typhimurium virulence plasmid gene rck. J. Clin. Invest. 90, 953-964. doi: 10.1172/JCI115972

Huttener, M., Prieto, A., Aznar, S., Bernabeu, M., Glaria, E., Valledor, A. F., et al. (2019). Expression of a novel class of bacterial Ig-like proteins is required for IncHI plasmid conjugation. PLoS Genet. 15:e1008399. doi: 10.1371/journal.pgen.1008399

Jones, G. W., Rabert, D. K., Svinarich, D. M., and Whitfield, H. J. (1982). Association of adhesive, invasive, and virulent phenotypes of Salmonella typhimurium with autonomous 60-megadalton plasmids. Infect. Immun. 38, 476-486. doi: 10.1128/IAI.38.2.476-486.1982

Lau, I. F., Filipe, S. R., Soballe, B., Okstad, O. A., Barre, F. X., and Sherratt, D. J. (2003). Spatial and temporal organization of replicating Escherichia coli chromosomes. Mol. Microbiol. 49, 731-743. doi: 10.1046/j.1365-2958.2003.03640.x

Lederberg, E. M. (1986). Plasmid prefix designations registered by the plasmid reference center 1977-1985. Plasmid 15, 57-92. doi: 10.1016/0147619X(86)90014-4

Lee, D. J., Bingle, L. E., Heurlier, K., Pallen, M. J., Penn, C. W., Busby, S. J., et al. (2009). Gene doctoring: a method for recombineering in laboratory and pathogenic Escherichia coli strains. BMC Microbiol. 9:252. doi: 10.1186/1471-2180-9-252

Lian, X., Wang, X., Liu, X., Xia, J., Fang, L., Sun, J., et al. (2019). oqxABpositive IncHI2 plasmid pHXY0908 increase Salmonella enterica serotype Typhimurium strains tolerance to ciprofloxacin. Front. Cell. Infect. Microbiol. 9:242. doi: 10.3389/fcimb.2019.00242

Lobato-Marquez, D., Moreno-Cordoba, I., Figueroa, V., Diaz-Orejas, R., and Garcia-del Portillo, F. (2015). Distinct type I and type II toxin-antitoxin modules control Salmonella lifestyle inside eukaryotic cells. Sci. Rep. 5:9374. doi: $10.1038 /$ srep09374

Mendoza, M., Herrero, A., and Rodicio, M. R. (2009). Evolutionary engineering in Salmonella: emergence of hybrid virulence-resistance plasmids in nontyphoid serotypes. Enferm. Infecc. Microbiol. Clin. 27, 37-43. doi: 10.1016/j. eimc.2008.09.001

Murray, G. L., Attridge, S. R., and Morona, R. (2005). Inducible serum resistance in Salmonella typhimurium is dependent on $w z z(f e p E)$-regulated very long O antigen chains. Microbes Infect. 7, 1296-1304. doi: 10.1016/j.micinf. 2005.04.015

Niki, H., and Hiraga, S. (1997). Subcellular distribution of actively partitioning F plasmid during the cell division cycle in E. coli. Cell 90, 951-957. doi: 10.1016/s0092-8674(00)80359-1

Paulsson, J. (2002). Multileveled selection on plasmid replication. Genetics 161, 1373-1384.

Pilla, G., and Tang, C. M. (2018). Going around in circles: virulence plasmids in enteric pathogens. Nat. Rev. Microbiol. 16, 484-495. doi: 10.1038/ s41579-018-0031-2

Raser, J. M., and O'Shea, E. K. (2005). Noise in gene expression: origins, consequences, and control. Science 309, 2010-2013. doi: 10.1126/ science. 1105891

Robinett, C. C., Straight, A., Li, G., Willhelm, C., Sudlow, G., Murray, A., et al. (1996). In vivo localization of DNA sequences and visualization of large-scale chromatin organization using lac operator/repressor recognition. J. Cell Biol. 135, 1685-1700. doi: 10.1083/jcb.135.6.1685

Rotger, R., and Casadesus, J. (1999). The virulence plasmids of Salmonella. Int. Microbiol. 2, 177-184.

Sanchez-Romero, M. A., and Casadesus, J. (2018). Contribution of SPI-1 bistability to Salmonella enterica cooperative virulence: insights from single cell analysis. Sci. Rep. 8:14875. doi: 10.1038/s41598-018-33137-z

Shapiro, H. M. (2003). Practical flow cytometry. Hoboken, New Jersey: John Wiley \& Sons. 
Suwandi, A., Galeev, A., Riedel, R., Sharma, S., Seeger, K., Sterzenbach, T., et al. (2019). Std fimbriae-fucose interaction increases Salmonella-induced intestinal inflammation and prolongs colonization. PLoS Pathog. 15:e1007915. doi: 10.1371/journal.ppat.1007915

Tinge, S. A., and Curtiss, R. 3rd. (1990). Conservation of Salmonella typhimurium virulence plasmid maintenance regions among Salmonella serovars as a basis for plasmid curing. Infect. Immun. 58, 3084-3092. doi: 10.1128/IAI.58.9. 3084-3092.1990

Urdaneta, V., Hernandez, S. B., and Casadesus, J. (2019). Mutational and non mutational adaptation of Salmonella enterica to the gall bladder. Sci. Rep. 9:5203. doi: 10.1038/s41598-019-41600-8

Vandenbosch, J. L., Rabert, D. K., Kurlandsky, D. R., and Jones, G. W. (1989). Sequence analysis of $r s k$, a portion of the 95-kilobase plasmid of Salmonella typhimurium associated with resistance to the bactericidal activity of serum. Infect. Immun. 57, 850-857. doi: 10.1128/IAI.57.3.850-857.1989

Veening, J. W., Smits, W. K., and Kuipers, O. P. (2008). Bistability, epigenetics, and bet-hedging in bacteria. Annu. Rev. Microbiol. 62, 193-210. doi: 10.1146/ annurev.micro.62.081307.163002

Volkmer, B., and Heinemann, M. (2011). Condition-dependent cell volume and concentration of Escherichia coli to facilitate data conversion for systems biology modeling. PLoS One 6:e23126. doi: 10.1371/journal.pone.0023126
Wang, X., Possoz, C., and Sherratt, D. J. (2005). Dancing around the divisome: asymmetric chromosome segregation in Escherichia coli. Genes Dev. 19, 2367-2377. doi: 10.1101/gad.345305

Weigel, W. A., and Dersch, P. (2018). Phenotypic heterogeneity: a bacterial virulence strategy. Microbes Infect. 20, 570-577. doi: 10.1016/j.micinf. 2018.01.008

Wong, N. C., Chatenay, D., Robert, J., and Poirier, M. G. (2010). Plasmid copy number noise in monoclonal populations of bacteria. Phys. Rev. 81:011909. doi: 10.1103/PhysRevE.81.011909

Conflict of Interest: The authors declare that the research was conducted in the absence of any commercial or financial relationships that could be construed as a potential conflict of interest.

Copyright (c) 2020 Sánchez-Romero, Mérida-Floriano and Casadesús. This is an open-access article distributed under the terms of the Creative Commons Attribution License (CC BY). The use, distribution or reproduction in other forums is permitted, provided the original author(s) and the copyright owner(s) are credited and that the original publication in this journal is cited, in accordance with accepted academic practice. No use, distribution or reproduction is permitted which does not comply with these terms. 